\title{
Infinite dimensional generalizations of Choi's Theorem
}

https://doi.org/10.1515/spma-2019-0006

Received March 8, 2019; accepted June 15, 2019

\begin{abstract}
In this paper we give a simple sequence of necessary and sufficient finite dimensional conditions for a positive map between certain subspaces of bounded linear operators on separable Hilbert spaces to be completely positive. These criterions are natural generalization of Choi's characterization for completely positive maps between pairs of linear operators on finite dimensional Hilbert spaces. We apply our conditions to a completely positive map between two trace class operators on separable Hilbert spaces. A completely positive map $\mu$ is called a quantum channel, if it is trace preserving, and $\mu$ is called a quantum subchannel if it decreases the trace of a positive operator. We give simple neccesary and sufficient condtions for $\mu$ to be a quantum subchannel. We show that $\mu$ is a quantum subchannel if and only if it has Hellwig-Kraus representation. The last result extends the classical results of Kraus and the recent result of Holevo for characterization of a quantum channel.
\end{abstract}

Keywords: Completely positive maps, Stinespring's dilation theorem, Choi's theorem, trace class operators, quantum channels, quantum subchannels, Hellwig-Kraus representation

MSC: 46N50, 81Q10, 94A40

\section{Introduction}

A quantum system in von Neumann model is described by a compact positive operator acting on a separable Hilbert $\mathcal{H}$ space with trace one. We denote by $T_{1}(\mathcal{H})$ the closed Banach space of trace class operators on $\mathcal{H}$. The evolution of an open quantum system from one system to another one can be described by a bounded positive linear operator $\mu$ between a pair of trace class operators of two separable Hilbert spaces $\mathcal{H}_{1}$ and $\mathcal{H}_{2}$ : $\mu: T_{1}\left(\mathcal{H}_{1}\right) \rightarrow T_{1}\left(\mathcal{H}_{2}\right)$. The standard assumption that $\mu$ is trace preserving. A more general assumption that $\mu$ decrease the trace of a positive operator [5]. It was realized by Kraus [8] that physical assumptions yield that $\mu$ has to be completely positive. A completely positive trace preserving $\mu$ is called a quantum channel. We call a completely positive $\mu$ which decreases the trace of positive operators a quantum subchannel. The purpose of this paper is threefold. First, we give a simple sequence of necessary and sufficient finite dimensional conditions for a positive map between certain subspaces of bounded linear operators on separable Hilbert spaces to be completely positive. These criterions are natural generalization of Choi's characterization for completely positive maps between pairs of linear operators on finite dimensional Hilbert spaces. Second, we give a simple sequence of necessary and sufficient finite dimensional conditions for that $\mu$ is a quantum subchannel. Third, we show that any quantum subchannel has Hellwig-Kraus representation. The last result extends the classical result of Kraus [8] and the recent result of Holevo [6] for characterization of a quantum channel.

We now explain in technical details the main results of this paper. The notion of a completely positive operator between two $C^{*}$-algebra was introduced by Stinespring [14]. In his seminal paper Stinespring gave ^Corresponding Author: Shmuel Friedland: Department of Mathematics, Statistics and Computer Science, University of
Illinois at Chicago, Chicago, Illinois 60607-7045, USA, E-mail: friedlan@uic.edu 
necessary and sufficient conditions for a positive map $\mu$ between two $C^{*}$ algebras to be completely positive. This is the celebrated Stinespring's dilation (representation) theorem. For the finite dimensional case, that is, the two $C^{*}$ algebras are the the spaces of square complex valued matrices, $\mu$ has a simple representation. However, given a linear transformation $\mu$ from one space of square complex valued matrices to another space of square complex matrices, it is not simple to decide if $\mu$ is completely positive. The simple necessary and sufficient conditions for $\mu$ to be completely positive were given by Choi in his fundamental paper [1]. In [5] the authors considered the evolution of an open quantum system. They showed that under corresponding quantum physics assumption the map $\mu$ on the trace class to itself has a special representation [5, (6)-(7)], which we call Hellwig-Kraus representation. (See (7) for the case $\mathcal{K}=\mathcal{H}$.) Kraus proved in [8, Theorem 3.3] that a quantum subchannel has representation (7) for $\mathcal{K}=\mathcal{H}$ assuming that $\mu$ is continuous with respect to ultraweak (weak operator) topology. Generalizations to quantum channels on a pair trace class operators were given by Holevo [6] and by Størmer to a von Neumann factor with a separating and cyclic vector [15].

In the first part of this paper we give a natural generalization of Choi's theorem for a bounded linear $\mu: \Sigma_{1} \rightarrow \Sigma_{2}$, where $\Sigma_{i}$ is given as a subspace of bounded linear operators $B\left(\mathcal{H}_{i}\right)$, endowed with the norm $\|\cdot\|_{i}$. (We assume that there exists a constant $K_{i}>0$ such that $\|g\| \leqslant K_{i}\|g\|_{i}$, where $\|g\|$ is the operator norm on $B\left(\mathcal{H}_{i}\right)$.) We impose the following conditions on $\Sigma_{i}$. The first condition is that each $\Sigma_{i}$ is invariant under the conjugation: $\Sigma_{i}^{*}=\Sigma_{i}$. The second condition is that each $\Sigma_{i}$ contains the subspace of all finite range operators. These two condition holds for all Schatten $p$-classes $T_{p}\left(\mathcal{H}_{i}\right)$ of compact operators for $p \in[1, \infty]$, where $T_{\infty}\left(\mathcal{H}_{i}\right)$ is the subspace of compact operators in $B\left(\mathcal{H}_{i}\right)$ with the standard operator norm. The third condition is more technical and is stated later. It is always satisfied if $\Sigma_{1}=T_{p}(\mathcal{H})$ for $p \in[1, \infty]$. Our main object is to give a sequence of necessary and sufficient conditions, where each condition is exactly the Choi conditions for the finite dimensional case.

Our first main result can be stated as follows. Assume that $\mathcal{H}$ is a Hilbert space with an inner product $\langle\cdot, \cdot\rangle$. For $\mathbf{v} \in \mathcal{H}$ and $a \in B(\mathcal{H})$ we denote by $\mathbf{v}^{\vee}$ and $a^{*} \in B(\mathcal{H})$ the bounded linear functional $\mathbf{v}^{\vee}(\mathbf{x})=\langle\mathbf{x}, \mathbf{v}\rangle$, and the adjoint operator $\langle a \mathbf{x}, \mathbf{y}\rangle=\left\langle\mathbf{x}, a^{*} \mathbf{y}\right\rangle$ on $\mathcal{H}$ respectively. For $\mathbf{u}, \mathbf{v} \in \mathcal{H}$ denote by $\mathbf{u v}^{\vee} \in B(\mathcal{H})$ the rank one operator $\mathbf{u v}^{\vee}(\mathbf{x})=\langle\mathbf{x}, \mathbf{v}\rangle \mathbf{u}$. Recall that a sequence $a_{n} \in B(\mathcal{H}), n \in \mathbb{N}$ converges in the weak operator topology to $a \in B(\mathcal{H})$ if $\lim _{n \rightarrow \infty}\left\langle a_{n} \mathbf{x}, \mathbf{y}\right\rangle=\langle a \mathbf{x}, \mathbf{y}\rangle$ for all $\mathbf{x}, \mathbf{y} \in \mathcal{H}$. Denote by $B(\mathcal{H})^{(n)}$ the space of $n \times n$ matrices $G=\left[g_{i j}\right]_{i=j=1}^{n}$, where $g_{i j} \in B(\mathcal{H})$ for $i, j \in[n]=\{1, \ldots, n\}$. Then $G^{*}$ is the adjoint matrix: $\left(G^{*}\right)_{i j}=$ $g_{j i}^{*}$. The matrix $G$ is selfadjoint if $G=G^{*}$. A matrix $G$ is called positive, which is denoted by $G \geqslant 0$, if $G$ is selfadjoint and $\sum_{i=j=1}^{n}\left\langle g_{i j} \mathbf{x}_{i}, \mathbf{x}_{j}\right\rangle \geqslant 0$ for all $\mathbf{x}_{1}, \ldots, \mathbf{x}_{n} \in \mathcal{H}$. Assume that $\mu: \Sigma_{1} \rightarrow \Sigma_{2}$ is a bounded linear map. Then $\mu$ is called completely positive if the following two conditions are satisfied: First, $\mu$ commutes with the adjoint map, i.e., $\mu(g)^{*}=\mu\left(g^{*}\right)$ for all $g \in \mathcal{H}_{1}$. (This is equivalent to the condition that $\mu$ preserves selfadjoint operators.) Second, for each $n \in \mathbb{N}$ and $G=\left[g_{i j}\right]_{i=j=1}^{n} \geqslant 0$ the matrix $\mu(G)=\left[\mu\left(g_{i j}\right)\right]_{i=j=1}^{n}$ is positive.

Our first generalization of Choi's result can be stated as follows:

Theorem 1. For $i=1,2$ assume that the following conditions hold:

1. $\mathcal{H}_{i}$ is a Hilbert space with an orthonormal basis $\left\{\mathbf{e}_{j, i}\right\}, j \in \mathbb{N}$.

2. For each $n \in \mathbb{N}$ denote by $P_{n, i}$ the projection on $\operatorname{span}\left(\mathbf{e}_{1, i}, \ldots, \mathbf{e}_{n, i}\right)$.

3. $\Sigma_{i}$ is a subspace of $B\left(\mathcal{H}_{i}\right)$ closed with respect to the norm $\|\cdot\|_{i}$ and the conjugation ${ }^{*}$. Furthermore $\Sigma_{i}$ contains the subspace of all finite range operators.

4. For each $g \in \Sigma_{1}$ the sequence $\mu\left(P_{n, 1} g P_{n, 1}\right), n \in \mathbb{N}$ converges in the weak operator topology to $\mu(g)$.

Then a bounded linear operator $\mu: \Sigma_{1} \rightarrow \Sigma_{2}$ is completely positive if and only if for each $n \in \mathbb{N}$ the matrix $L_{n}(\mu):=\left[P_{n, 2} \mu\left(\mathbf{e}_{i, 1} \mathbf{e}_{j, 1}^{\vee}\right) P_{n, 2}\right]_{i=j=1}^{n} \in B\left(\Sigma_{2}\right)^{(n)}$ is positive.

We will show that if $\Sigma_{1}=T_{p}\left(\mathcal{H}_{1}\right)$ then the condition (4) is satisfied. In other cases the assumption (4) is satisfied if $\mu$ maps any convergent sequence in the strong operator topology to a convergent sequence in the weak operator topology. A stronger condition than (4) is that $\mu$ is normal. That is, if $a_{n} \in \Sigma_{1}, n \in \mathbb{N}$ is a sequence of increasing positive operators that converges to $a \in \Sigma_{1}$ in the weak operator topology, then $\mu\left(a_{n}\right), n \in \mathbb{N}$ is an increasing sequence that converges to $\mu(a)$ in the weak operator topology. 
Using a standard model of an interaction of an open quantum system with another quantum system Hellwig and Kraus [5, (6)-(7)] showed that $\mu$ has the following form: $\mu(a)=\sum_{i=1}^{\infty} A_{i} a A_{i}^{\vee}$. Here we assume that $A_{i} \in L\left(\mathcal{H}_{1}, \mathcal{H}_{2}\right), A_{i}^{\vee}: \mathcal{H}_{2}^{\vee} \rightarrow \mathcal{H}_{1}^{\vee}$, (the dual operator), for $i \in \mathbb{N}$. Furthermore we have the inequality $\sum_{i=1}^{\infty} A_{i}^{\vee} A_{i} \leqslant I d$. To be precise it is assumed in [5] that $\mathcal{H}_{1}=\mathcal{H}_{2}$. We call such $\mu$ a quantum subchannel. It is straightforward to show that a quantum subchannel is a quantum channel if and only iff $\sum_{i=1}^{\infty} A_{i}^{\vee} A_{i}=I d$. It was shown by Kraus [8, Theorem 3.3] that assuming that $\mathcal{H}_{1}=\mathcal{H}_{2}$ and $\mu$ continuous with respect the weak operator topology a quantum subchannel has the above representation. Our second main result is that a subchannel has the above Hellwig-Kraus representation (without the assumption that $\mu$ is continuous with respect to the weak operator topology). For a quantum channel this result was shown by Holevo [6]. (In this case $\sum_{i=1}^{\infty} A_{i}^{\vee} A_{i}=I d$, and the above representation is called Kraus representation.)

We now survey briefly the contents of this paper. In $\S 2$ we recall the Stinespring dilatation theorem [14] and Choi's theorem on characterization of completely positive operators between two spaces of complex square matrices [1]. In $\S 3$ we state Theorem 4, which is a variation of Theorem 1 . We then prove the two theorems. In $\S 4$ we show that for $\Sigma_{1}=T_{p}\left(\mathcal{H}_{1}\right)$ the condition (4) of Theorem 1 is satisfied. In $\S 5$ we discuss the notions of quantum channels and subchannels on finite and infinite dimensional subspaces. We show that any quantum subchannel has Hellwig-Kraus representation.

\section{Stinespring and Choi theorems}

Let $\mathcal{A}$ be a $C^{*}$-algebra and denote by $K(\mathcal{A})$ the cone of positive operators. So $0 \leqslant a \Longleftrightarrow a \in K(\mathcal{A}) \Longleftrightarrow a=$ $b b^{*}$. Assume that $\mathcal{B}$ is another $C^{*}$ algebra. A bounded linear map $\mu: \mathcal{A} \rightarrow \mathcal{B}$ is called positive, and denote by $\mu \geqslant 0$, if $\mu(a) \geqslant 0$ for each $a \geqslant 0$. A completely positive operator $\mu$ was defined by Stinespring [14] as follows: Let $\mathcal{A}^{(n)}$ be the algebra of $n \times n$ matrices $G=\left[g_{i j}\right]_{i=j=1}^{n}$, where $g_{i j} \in \mathcal{A}$ for $i, j=[n]=\{1, \ldots, n\}$. One can show that $\mathcal{A}^{(n)}$ is a $C^{*}$-algebra, where $\left(G^{*}\right)_{i j}=g_{j i}^{*}, i, j \in[n]$. Indeed, use the Gelfand-Naimark theorem [3] to assume that $\mathcal{A}$ is a $C^{*}$-algebra of bounded operators on a Hilbert space $\mathcal{H}$. Then $\mathcal{A}^{(n)}$ represents the $C^{*}$-algebra of bounded operators on the direct sum of $n$ copies of $\mathcal{H}$. Clearly, $\mu$ induces the linear bounded maps $\mu_{n}: \mathcal{A}^{(n)} \rightarrow \mathcal{B}^{(n)}$ for each $n \in \mathbb{N}$. The map $\mu$ is completely positive if $\mu_{n} \geqslant 0$ for each $n \in \mathbb{N}$.

Let $\mathcal{K}$ be a Hilbert space and denote by $B(\mathcal{K})$ the $C^{*}$-algebra of bounded linear operators $g: \mathcal{K} \rightarrow \mathcal{K}$. Assume that $\mathcal{A}$ is a $C^{*}$-algebra with the unit 1 . The $\rho: \mathcal{A} \rightarrow B(\mathcal{K})$ is called a ${ }^{*}$-representation if $\rho$ is positive and $\rho(1)=I d$.

Assume $\mathcal{A}$ is a unital $C^{*}$ algebra, $\mathcal{H}$ is a Hilbert space and $\mu: \mathcal{A} \rightarrow B(\mathcal{H})$ is a bounded linear operator. The Stinespring dilation theorem says that there exists a *-representation $\rho: \mathcal{A} \rightarrow B(\mathcal{K})$ such that $\mu=V^{*} \rho V$ for a some bounded operator $V: \mathcal{H} \rightarrow \mathcal{K}$ if and only $\mu$ is completely positive.

One can drop the assumption that $\mathcal{A}$ is unital as follows. Suppose that $\mathcal{A}$ is not unital and $\mu: \mathcal{A} \rightarrow B(\mathcal{H})$ is completely positive. Then $\mathcal{A}$ is contained in a unital $C^{*}$-algebra $\mathcal{A}_{1}=\mathcal{A} \oplus \mathbb{C}$ with naturally defined operations [2, Proposition I.1.3]. Furthermore, $\mu$ lifts to a completely positive $\mu_{1}: \mathcal{A}_{1} \rightarrow B(\mathcal{H})$, where $\mu_{1}((a, z))=\mu(a)$. We call $\rho: \mathcal{A} \rightarrow B(\mathcal{K})$ a representation, if $\rho$ is the restriction of a representation $\rho_{1}: \mathcal{A}_{1} \rightarrow B(\mathcal{K})$. We thus obtain the following theorem, which is usually called Stinespring's representation theorem [10, Theorem 6.1.1]:

Theorem 2. Let $\mu$ be a completely positive map from a $C^{*}$-algebra $\mathcal{A}$ into $B(\mathcal{H})$. Then there is a representation $\rho$ of $\mathcal{A}$ on some Hilbert space $\mathcal{K}$ and a bounded linear operator $V: \mathcal{H} \rightarrow \mathcal{K}$ such that $\mu(a)=V^{*} \rho(a) V$. If $\mathcal{A}$ has a unit 1 and $\mu(1)=I d$ then $V$ is an isometry.

Assume that $\mathcal{H}$ and $\mathcal{K}$ are separable Hilbert space, i.e. $\mathcal{H}$ and $\mathcal{K}$ have countable orthonormal bases. Let $\mu$ : $B(\mathcal{K}) \rightarrow B(\mathcal{H})$ be a bounded linear map. What are simple necessary and sufficient conditions for $\mu$ to be completely positive?

When $\mathcal{K}$ and $\mathcal{H}$ are finite dimensional, the elegant necessary and sufficient conditions were given by Choi [1]: Choose orthonormal bases $\mathbf{k}_{1}, \ldots, \mathbf{k}_{n}$ and $\mathbf{h}_{1}, \ldots, \mathbf{h}_{m}$ in $\mathcal{K}$ and $\mathcal{H}$ respectively. This will give an isomorphism $\iota_{\mathcal{K}}: B(\mathcal{K}) \rightarrow \mathbb{C}^{n \times n}, \iota_{\mathcal{H}}: B(\mathcal{H}) \rightarrow \mathbb{C}^{m \times m}$. More precisely, for each $\mathbf{0} \neq \mathbf{u}, \mathbf{v} \in \mathcal{K}$ denote by 
$\mathbf{u v}^{\vee}$ the following rank one operator in $B(\mathcal{K}):\left(\mathbf{u v}^{\vee}\right)(\mathbf{w})=\langle\mathbf{w}, \mathbf{v}\rangle \mathbf{u}$, where $\langle\cdot, \cdot\rangle$ is the inner product in $\mathcal{K}$. So $\mathbf{k}_{i} \mathbf{k}_{j}^{\vee}, i, j \in[n]$ is the standard basis of $B(\mathcal{K})$ associated with the orthonormal basis of $\mathbf{k}_{1}, \ldots, \mathbf{k}_{n}$, which corresponds to the standard basis on $n \times n$ matrices $\mathbb{C}^{n \times n}$. Then $\mu$ is completely positive if and only if the hermitian matrix of order $m n$, written in a block matrix form, is positive definite:

$$
L(\mu)=\left[\mu\left(\mathbf{k}_{i} \mathbf{k}_{j}^{\vee}\right)\right]_{i=j=1}^{n} \geqslant 0 .
$$

See [16, Chapter 2.2] for more details on Kraus decomposition, the Stinespring dilatation, and the Choi theorem on finite dimensional inner product spaces.

\section{Proof of the main theorem}

Let $\mathcal{H}$ be a Hilbert space and assume $\left\{g_{n}\right\}_{n \in \mathbb{N}} \subset B(\mathcal{H})$. Then $\lim _{n \rightarrow \infty} g_{n}=g$ :

1. in the strong operator topology, abbreviated as strongly convergent sequence, if $\lim _{n \rightarrow \infty} g_{n} \mathbf{x}=g \mathbf{x}$ for each $\mathbf{x} \in \mathcal{H}$;

2. in the weak operator topology, abbreviated as weakly convergent sequence, if $\lim _{n \rightarrow \infty}\left\langle g_{n} \mathbf{x}, \mathbf{y}\right\rangle=\langle g \mathbf{x}, \mathbf{y}\rangle$ for each $\mathbf{x}, \mathbf{y} \in \mathcal{H}$.

Assume that $\mathcal{K}$ is another Hilbert space, and let $\mu: B(\mathcal{K}) \rightarrow B(\mathcal{H})$ be a bounded linear map. We say that $\mu$ maps strongly convergent sequences to weakly convergent sequences if for each strongly convergent sequence $\lim _{n \rightarrow \infty} g_{n}=g$ we have that $\lim _{n \rightarrow \infty} \mu\left(g_{n}\right)=\mu(g)$ in the weak operator topology.

Recall the following well known result, whose proof we leave to the reader.

Lemma 3. Let $\mathcal{H}$ be a Hilbert space with an infinite countable basis $\left\{\mathbf{h}_{n}\right\}, n \in \mathbb{N}$. Denote by $P_{n}: \mathcal{H} \rightarrow \mathcal{H}$ the orthogonal projection on $\operatorname{span}\left(\mathbf{h}_{1}, \ldots, \mathbf{h}_{n}\right)$ for $n \in \mathbb{N}$. Then for each $\mathbf{x} \in \mathcal{H}$ and $g \in B(\mathcal{H})$

$$
\lim _{n \rightarrow \infty}\left\|P_{n} \mathbf{x}-\mathbf{x}\right\|=\lim _{n \rightarrow \infty}\left\|g P_{n} \mathbf{x}-g \mathbf{x}\right\|=\lim _{n \rightarrow \infty}\left\|P_{n} g P_{n} \mathbf{x}-g \mathbf{x}\right\|=0 .
$$

The above lemma is equivalent to the statement:

$$
\lim _{n \rightarrow \infty} P_{n}=I d, \quad \lim _{n \rightarrow \infty} g P_{n}=\lim _{n \rightarrow \infty} P_{n} g P_{n}=g
$$

in the strong operator topology.

To prove Theorem 1 it will be convenient to state a variation of Theorem 1:

Theorem 4. Let $\mathcal{K}$ and $\mathcal{H}$ be Hilbert spaces with countable bases $\mathbf{k}_{1}, \ldots, \mathbf{h}_{1}, \ldots$, respectively. For each $m \in \mathbb{N}$ denote by $P_{m}$ the orthogonal projection of $\mathcal{H}$ on $\operatorname{span}\left(\mathbf{h}_{1}, \ldots, \mathbf{h}_{m}\right)$ if $\operatorname{dim} \mathcal{H} \geqslant m$. Assume that $\mu: B(\mathcal{K}) \rightarrow B(\mathcal{H})$ is a bounded linear operator which maps strongly convergent sequences to weak convergent sequences. Then in each of the following cases $\mu$ is completely positive if and only if the corresponding conditions hold:

1. Assume that $\mathcal{K}$ has dimension $n$ with an orthonormal basis $\mathbf{k}_{1}, \ldots, \mathbf{k}_{n}$ and $\mathcal{H}$ is infinite dimensional. Then for each $m \in \mathbb{N}$ the hermitian matrix $L_{n, m}(\mu):=\left[P_{m} \mu\left(\mathbf{k}_{i} \mathbf{k}_{j}^{\vee}\right) P_{m}\right]_{i=j=1}^{n} \in B(\mathcal{H})^{(n)}$ is positive.

2. Assume that $\mathcal{H}$ has dimension $m$ with an orthonormal basis $\mathbf{h}_{1}, \ldots, \mathbf{h}_{m}$ and $\mathcal{K}$ is infinite dimensional. Then for each $n \in \mathbb{N}$ the the matrix $L_{n, m}(\mu):=\left[\mu\left(\mathbf{k}_{i} \mathbf{k}_{j}^{\vee}\right)\right]_{i=j=1}^{n} \in B(\mathcal{H})^{(n)}$ is positive.

3. Assume that $\mathcal{K}$ and $\mathcal{H}$ are infinite dimensional. Then for each $n \in \mathbb{N}$ the matrix $L_{n}(\mu):=$ $\left[P_{n} \mu\left(\mathbf{k}_{i} \mathbf{k}_{j}^{\vee}\right) P_{n}\right]_{i=j=1}^{n} \in B(\mathcal{H})^{(n)}$ is positive.

Note that if $\mathcal{K}$ is finite dimensional then the norm operator topology, the strong operator topology and the weak are operator topology on $B(\mathcal{K})$ are same topologies.

Proof. Assume that $\mu$ is completely positive. Suppose that $\operatorname{dim} \mathcal{K} \geqslant n$. Let $G=\left[\mathbf{k}_{i} \mathbf{k}_{j}^{\vee}\right]_{i, j=1}^{n}$. As $G=$ $\left(\frac{1}{\sqrt{n}} G\right)\left(\frac{1}{\sqrt{n}} G\right)^{*}$ it follows that $G$ is positive. Hence $\mu_{n}(G)=\left[\mu\left(\mathbf{k}_{i} \mathbf{k}_{j}^{\vee}\right)\right]_{i, j=1}^{n}$ is positive. In particular,

$$
\sum_{i=j=1}^{n}\left\langle\mu\left(\mathbf{k}_{i} \mathbf{k}_{j}^{\vee}\right) \mathbf{x}_{j}, \mathbf{x}_{i}\right\rangle \geqslant 0
$$


for each $\mathbf{x}_{1}, \ldots, \mathbf{x}_{n} \in \mathcal{H}$. Assume that $\operatorname{dim} \mathcal{H} \geqslant m$. Then $P_{m} \mathbf{x}_{1}, \ldots, P_{m} \mathbf{x}_{n} \in \operatorname{span}\left(\mathbf{h}_{1}, \ldots, \mathbf{h}_{m}\right)$. Therefore $L_{n, m}(\mu)$, as defined in part (1) is positive. In particular, if $\operatorname{dim} \mathcal{H} \geqslant n$ we obtain that $L_{n}(\mu)=L_{n, n}(\mu)$ is positive.

We now need to show the sufficiency of the conditions in (1), (2) and (3).

(1) We first show that $M_{n}(\mu):=\left[\mu\left(\mathbf{k}_{i} \mathbf{k}_{j}^{\vee}\right)\right]_{i=j=1}^{n} \in B(\mathcal{H})^{(n)}$ is positive. Fix $\mathbf{x}_{1}, \ldots, \mathbf{x}_{n} \in \mathcal{H}$. As $L_{n, m}(\mu) \geqslant 0$ it follows that

$$
\sum_{p=q=1}^{n}\left\langle P_{m} \mu\left(\mathbf{k}_{p} \mathbf{k}_{q}^{\vee}\right) P_{m} \mathbf{x}_{q}, \mathbf{x}_{p}\right\rangle \geqslant 0 .
$$

Let $m \rightarrow \infty$ and use Lemma 3 to deduce that $\sum_{p=q=1}^{n}\left\langle\mu\left(\mathbf{k}_{p} \mathbf{k}_{q}^{\vee}\right) \mathbf{x}_{q}, \mathbf{x}_{p}\right\rangle \geqslant 0$. Hence $M_{n}(\mu) \geqslant 0$.

We now show that the image of $B(\mathcal{K})^{(k)}$ by $\mu_{k}$ is positive for each $k \in \mathbb{N}$. Recall that $B(\mathcal{K})$ is isomorphic to $\mathbb{C}^{n \times n}$. Hence $B(\mathcal{K})^{(k)}$ is isomorphic to $\left(\mathbb{C}^{n \times n}\right)^{(k)}=\mathbb{C}^{(k n) \times(k n)}$. Denote by $\tilde{\mu}_{k}: \mathbb{C}^{(k n) \times(k n)} \rightarrow B(\mathcal{H})^{(k)}$ the induced map by $\mu_{k}$. To show that $\tilde{\mu}_{k}$ is positive, it is enough to show that the image of rank one $(k n) \times(k n)$ matrices positive matrix by $\tilde{\mu}_{k}$ is positive. A rank one positive matrix of order $k n$ in the block form is $U=\left[\mathbf{u}_{i} \mathbf{u}_{j}^{*}\right]_{i=j=1}^{k}$, where $\mathbf{u}_{1}=\left(u_{1,1}, \ldots, u_{n, 1}\right)^{\top}, \ldots, \mathbf{u}_{k}=\left(u_{1, k}, \ldots, u_{n, k}\right)^{\top} \in \mathbb{C}^{n}$. This rank one matrix is represented by $V=$ $\left[\mathbf{v}_{i} \mathbf{v}_{j}^{\vee}\right]_{i=j=1}^{k} \in B(\mathcal{K})^{(k)}$, where $\mathbf{v}_{i}=\sum_{p=1}^{n} u_{p, i} \mathbf{k}_{p}$ for $i \in[k]$.

A straightforward computation yields:

$$
F=\left[F_{i j}\right]_{i=j=1}^{k}=\mu_{k}\left(\left[\mathbf{v}_{i} \mathbf{v}_{j}^{\vee}\right]_{i=j=1}^{k}\right)=\left[\mu\left(\mathbf{v}_{i} \mathbf{v}_{j}^{\vee}\right)\right]_{i=j=1}^{k}=\left[\mathbf{u}_{i}^{\top} M_{n}(\mu) \overline{\mathbf{u}}_{j}\right]_{i=j=1}^{k} \in B(\mathcal{H})^{(k)}
$$

is selfadjoint. Assume that $\mathbf{x}_{1}, \ldots, \mathbf{x}_{k} \in \mathcal{H}$. Then

$$
\sum_{i=j=1}^{k}\left\langle F_{i j} \mathbf{x}_{j}, \mathbf{x}_{i}\right\rangle=\sum_{p=q=1}^{n}\left\langle\mu\left(\mathbf{k}_{p} \mathbf{k}_{q}^{\vee}\right) \mathbf{y}_{q}, \mathbf{y}_{p}\right\rangle, \quad \mathbf{y}_{q}=\sum_{j=1}^{k} \bar{u}_{q, j} \mathbf{x}_{j}, q \in[n] .
$$

Hence $F$ is positive.

(ii) We need to show that $\mu_{k}$ is positive for each $k \in \mathbb{N}$. Assume that $G=\left[g_{i j}\right]_{i=j=1}^{k} \in B(\mathcal{K})^{(k)}$ is positive. Denote by $Q_{n}$ the orthogonal projection of $\mathcal{K}$ on $\operatorname{span}\left(\mathbf{k}_{1}, \ldots, \mathbf{k}_{n}\right)$. Let $\mathcal{K}_{n}=Q_{n} \mathcal{K}$. Define $G_{n}=\left[Q_{n} g_{i j} Q_{n}\right]_{i=j=1}^{k}$. Then $G_{n} \geqslant 0$. Observe that $G_{n} \in B\left(\mathcal{K}_{n}\right)^{(k)}$. The assumption that $L_{n, m}(\mu):=\left[\mu\left(\mathbf{k}_{i} \mathbf{k}_{j}^{\vee}\right)\right]_{i=j=1}^{n} \in B(\mathcal{H})^{(n)}$ is positive and Choi's theorem yield that $\mu_{k}\left(G_{n}\right) \geqslant 0$. By Lemma $3 Q_{n} g Q_{n}, n \in \mathbb{N}$ converges strongly to $g$ for each $g \in B(\mathcal{K})$. As $\mu\left(Q_{n} g Q_{n}\right), n \in \mathbb{N}$ converges weakly to $\mu(g)$ it follows that for fixed $\mathbf{x}_{1}, \ldots, \mathbf{x}_{k} \in \mathcal{K}$ we have the equaity

$$
\sum_{i=j=1}^{k}\left\langle\mu\left(g_{i j}\right) \mathbf{x}_{j}, \mathbf{x}_{i}\right\rangle=\lim _{n \rightarrow \infty} \sum_{i=j=1}^{k}\left\langle\mu\left(Q_{n} g_{i j} Q_{n}\right) \mathbf{x}_{j}, \mathbf{x}_{i}\right\rangle \geqslant 0 .
$$

Hence $\mu_{k}(G)$ is positive.

(iii) Assume that for each $n \in \mathbb{N}$ the matrix $L_{n}(\mu):=\left[P_{n} \mu\left(\mathbf{k}_{i} \mathbf{k}_{j}^{\vee}\right) P_{n}\right]_{i=j=1}^{n} \in B(\mathcal{H})^{(n)}$ is positive. Fix $m \in \mathbb{N}$ and let $\mathcal{H}_{m}=P_{m} \mathcal{H}_{m}$. It is straightforward to show that for each $n \geqslant m$ the matrix $L_{n, m}(\mu) \in B\left(\mathcal{H}_{m}\right)^{(n)}$, as defined in (1), is positive. Let $\hat{\mu}_{m}: B(\mathcal{K}) \rightarrow B\left(\mathcal{H}_{m}\right)$ be given by $\hat{\mu}_{m}(g)=P_{m} \mu(g) P_{m}$. Part (2) yields that $\hat{\mu}_{m}$ is completely positive. That is for each $k \in \mathbb{N}$ and positive $G=\left[g_{i j}\right]_{i=j=1}^{k} \in B(\mathcal{K})^{(k)}$ the matrix $\left[\hat{\mu}\left(g_{i j}\right)\right]_{i=j=1}^{k}=$ $\left[P_{m} \mu\left(g_{i j}\right) P_{m}\right]_{i=j=1}^{k} \in B\left(\mathcal{H}_{m}\right)^{(k)}$ is positive. That is , for fixed $\mathbf{x}_{1}, \ldots, \mathbf{x}_{k} \in \mathcal{H}$ we have

$$
\sum_{i=j=1}^{k}\left\langle P_{m} \mu\left(g_{i j}\right) P_{m}\left(P_{m} \mathbf{x}_{j}\right), P_{m} \mathbf{x}_{i}\right\rangle=\sum_{i=j=1}^{k}\left\langle P_{m} \mu\left(g_{i j}\right) P_{m} \mathbf{x}_{j}, \mathbf{x}_{j}\right\rangle \geqslant 0 .
$$

Let $m \rightarrow \infty$ and use Lemma 3 to deduce that $\mu_{k}(G) \geqslant 0$. Thus $\mu_{k}$ is positive for each $k \in \mathbb{N}$.

Proof of Theorem 1. Since $\Sigma_{1}$ and $\Sigma_{2}$ contain all finite range operators the proof of Theorem 4 yields that the condition that $L_{m}(\mu)=\left[P_{m, 2} \mu\left(\mathbf{e}_{i, 1} \mathbf{e}_{j, 1}^{\vee}\right) P_{m, 2}\right]_{i=j=1}^{n} \in B\left(\Sigma_{2}\right)^{(n)}$ is positive is necessary. It is left to show that this condition is sufficient. Fix $n \in \mathbb{N}$ and let $\mathcal{K}_{n}=P_{n, 1} \mathcal{H}_{1}$. Let $\mu_{n}^{\prime}$ be the restriction of $\mu$ to $B\left(\mathcal{K}_{n}\right)$. Assume that $m \geqslant n$. The assumption that $L_{m}(\mu)$ positive yields that $L_{n, m}(\mu)=\left[P_{m, 2} \mu\left(\mathbf{e}_{i, 1} \mathbf{e}_{j, 1}^{\vee}\right) P_{m, 2}\right]_{i, j=1}^{n} \in B\left(\Sigma_{2}\right)^{(n)}$ is positive. Use the prove of part (1) of Theorem 4 to deduce that $\mu_{n}^{\prime}$ is completely positive. Next, fix $m \in \mathbb{N}$ let $\mathcal{H}_{m}=P_{m, 2} \mathcal{H}_{2}$ and $\hat{\mu}_{m}: \Sigma_{1} \rightarrow B\left(\mathcal{H}_{m}\right)$ be defined as in the proof of part (3) of Theorem 4. As $\mu\left(P_{n, 1} g P_{n, 1}\right), n \in \mathbb{N}$ converges weakly to $\mu(g)$ the proof of part (2) of Theorem 4 yields that $\hat{\mu}_{m}$ is completely positive. The proof of part (3) of Theorem 4 yields the proof of Theorem 1. 


\section{Additional results}

We now recall some standard facts about bounded linear operators on a Hilbert space [9]. Let $\mathcal{H}$ be a Hilbert space with the norm $\|\mathbf{x}\|=\sqrt{\langle\mathbf{x}, \mathbf{x}\rangle}$. For $g \in B(\mathcal{H})$ let $\|\mathbf{g}\|=\sup \{\|B \mathbf{x}\|,\|\mathbf{x}\| \leqslant 1\}$ be the operator norm of g. Denote by $T_{\infty}(\mathcal{H}) \subset B(\mathcal{H})$ the closed subspace of compact operators on $\mathcal{H}$. Recall that $g \in T_{\infty}(\mathcal{H})$ has a convergent singular value decomposition with respect to the operator norm $\|\cdot\|_{\infty}:=\|\cdot\|$ :

$$
\begin{aligned}
g & =\sum_{i=1}^{\infty} \sigma_{i}(g) \mathbf{k}_{i} \mathbf{h}_{i}^{\vee},\left\langle\mathbf{k}_{i}, \mathbf{k}_{j}\right\rangle=\left\langle\mathbf{h}_{i}, \mathbf{h}_{j}\right\rangle=\delta_{i j}, i, j \in \mathbb{N}, \\
\|g\| & =\sigma_{1}(g) \geqslant \cdots \geqslant \sigma_{n}(g) \geqslant \cdots \geqslant 0, \lim _{n \rightarrow \infty} \sigma_{n}(g)=0 .
\end{aligned}
$$

In particular, $\left\|g-\sum_{i=1}^{n} \sigma_{i}(g) \mathbf{k}_{i} \mathbf{h}_{i}^{\vee}\right\|=\sigma_{n+1}(g)$. Furthermore

$$
g^{*}=\sum_{i=1}^{\infty} \sigma_{i}(g) \mathbf{h}_{i} \mathbf{k}_{i}^{\vee}, \quad \sqrt{g g^{*}}=\sum_{i=1}^{\infty} \sigma_{i}(g) \mathbf{k}_{i} \mathbf{k}_{i}^{\vee} \geqslant 0, \quad \sqrt{g^{*} g}=\sum_{i=1}^{\infty} \sigma_{i}(g) \mathbf{h}_{i} \mathbf{h}_{i}^{\vee} \geqslant 0 .
$$

For $p \in[1, \infty)$ denote by

$$
T_{p}(\mathcal{H})=\left\{g \in T_{\infty}(\mathcal{H}),\|g\|_{p}:=\left(\sum_{i=1}^{\infty} \mid \sigma_{i}(g)^{p}\right)^{\frac{1}{p}}<\infty\right\}
$$

the $p$-Schatten class $[12,13]$. In particular for $g \in T_{p}(\mathcal{H})$ we have the equalities

$$
\begin{array}{r}
\left\|g-\sum_{i=1}^{n} \sigma_{i}(g) \mathbf{k}_{i} \mathbf{h}_{i}^{\vee}\right\|_{p}=\left(\sum_{j=n+1}^{\infty} \sigma_{j}(g)^{p}\right)^{\frac{1}{p}}, n \in \mathbb{N}, \\
\left\|\sqrt{g g^{*}}\right\|_{p}=\left\|\sqrt{g^{*} g}\right\|_{p}=\|g\|_{p}=\left\|g^{*}\right\|_{p}, \quad p \in[1, \infty) .
\end{array}
$$

Recall that if $a \in B(\mathcal{H})$ and $g \in T_{p}(\mathcal{H})$ then $a g$, $g a \in T_{p}(\mathcal{H})$. Furthermore

$$
\max \left(\|a g\|_{p},\|g a\|_{p}\right) \leqslant\|a\|\|g\|_{p}, \quad a \in B(\mathcal{H}), g \in T_{p}(\mathcal{H}), p \in[1, \infty] .
$$

The classes $T_{1}(\mathcal{H})$ and $\mathcal{T}_{2}(\mathcal{H})$ are called the trace class and the Hilbert-Schmidt class respectively.

The following result is well known and we bring its proof for completeness:

Lemma 5. Let $\mathcal{H}$ be a countable Hilbert space with an orthonormal basis $\left\{\mathbf{e}_{i}\right\}, i \in \mathbb{N}$. Let $P_{n} \in B(\mathcal{H})$ be the orthonormal projection on $\operatorname{span}\left(\mathbf{e}_{1}, \ldots, \mathbf{e}_{n}\right)$ for $n \in \mathbb{N}$. Assume that $p \in[1, \infty]$ and $g \in T_{p}(\mathcal{H})$. Then $\lim _{n \rightarrow \infty}\left\|P_{n} g P_{n}-g\right\|_{p}=0$.

Proof. Assume $g$ has the singular value decomposition (2). Let $g_{m}=\sum_{i=1}^{m} \sigma_{i}(g) \mathbf{k}_{i} \mathbf{h}_{i}^{\vee}$. Fix $\varepsilon>0$. Choose $M(\varepsilon)$ such that $\left\|g-g_{m}\right\|_{p}<\frac{\varepsilon}{3}$. Clearly

$$
P_{n} g P_{n}-g=\left(P_{n} g_{m} P_{n}-g_{m}\right)+P_{n}\left(g-g_{m}\right) P_{n}+\left(g_{m}-g\right) .
$$

Hence

$$
\left\|P_{n} g P_{n}-g\right\|_{p} \leqslant\left\|P_{n} g_{m} P_{n}-g_{m}\right\|_{p}+\left\|P_{n}\left(g-g_{m}\right) P_{m}\right\|_{p}+\left\|g_{m}-g\right\|_{p} .
$$

As $\left\|P_{n}\right\|=1$ it follows that

$$
\left\|P_{n}\left(g-g_{m}\right) P_{m}\right\|_{p} \leqslant\left\|P_{n}\right\|\left\|g-g_{m}\right\|_{p}\left\|P_{m}\right\| \leqslant\left\|g-g_{m}\right\|_{p}<\varepsilon / 3 .
$$

Observe next that for $\mathbf{u} \in \mathcal{H}$ we have that $\mathbf{u}^{\vee} P_{n}=\left(P_{n} \mathbf{u}\right)^{\vee}$. Lemma 3 yields that

$$
\lim _{n \rightarrow \infty}\left\|P_{n} \mathbf{k}_{i}-\mathbf{k}_{i}\right\|=\lim _{n \rightarrow \infty}\left\|\left(P_{n} \mathbf{h}_{i}\right)^{\vee}-\mathbf{h}_{i}^{\vee}\right\|=0 \text { for } i \in \mathbb{N} .
$$

Hence $\lim _{n \rightarrow \infty}\left\|P_{n}\left(\mathbf{k}_{i} \mathbf{h}_{i}^{\vee}\right) P_{n}-\mathbf{k}_{i} \mathbf{h}_{i}^{\vee}\right\|=0$ for each $i \in \mathbb{N}$. In particular $\lim _{n \rightarrow \infty}\left\|P_{n} g_{m} P_{n}-g_{m}\right\|=0$. Therefore $\lim _{n \rightarrow \infty} \sigma_{i}\left(P_{n} g_{m} P_{n}-g_{m}\right)=0$ for each $i \in \mathbb{N}$. As the dimension of the range of $g_{m}$ and $P_{n} g_{m} P_{n}$ is at most $m$ it follows that $\sigma_{i}\left(P_{n} g_{m} P_{n}-g_{m}\right)=0$ for $i>2 m$. Hence there exists $N(\varepsilon) \in \mathbb{N}$ such that $\left\|P_{n} g_{m} P_{n}-g_{m}\right\|_{p}<\varepsilon / 3$ for $n>N(\varepsilon)$. In particular, $\left\|P_{n} g P_{n}-g\right\|_{p}<\varepsilon$. 
Corollary 6. Let $\mathcal{K}$ be an infinite dimensional separable Hilbert space, and $\Sigma_{2}$ be a Banach space. Let $P_{n}$ : $\mathcal{K} \rightarrow \mathcal{K}$ be the orthogonal projection on $\operatorname{span}\left(\mathbf{k}_{1}, \ldots, \mathbf{k}_{n}\right)$ for $n \in \mathbb{N}$. Assume that $\mu: T_{p}(\mathcal{K}) \rightarrow \Sigma_{2}, p \in[1, \infty]$ is a bounded linear operator. Then for each $g \in T_{p}(\mathcal{K}) \lim _{n \rightarrow \infty}\left\|\mu\left(P_{n} g P_{n}\right)-\mu(g)\right\|=0$.

Assume that in Theorem $1 \Sigma_{1}=T_{p}\left(\mathcal{H}_{1}\right)$ for some $p \in[1, \infty]$. Then the assumption (4) holds.

We now give a well known condition which ensures the condition (4) on $\mu$ in Theorem 1 is satisfied. Let $S(\mathcal{K}) \subset B(\mathcal{K})$ be the real subspace of selfadjoint operators on the Hilbert space $\mathcal{K}$. Let $K(B(\mathcal{K})) \subset S(\mathcal{K})$ be the cone of positive operators. Then $K(B(\mathcal{K}))$ is a closed, pointed $(K(B(\mathcal{K})) \cap(-K(B(\mathcal{K})))=\{0\}$, generating $(S(\mathcal{K})=K(B(\mathcal{K}))-K(B(\mathcal{K})))$ cone with interior. For $a, b \in S(\mathcal{K})$ denote by $a \geqslant b$ if $a-b \geqslant 0(a-b \in K(B(\mathcal{K})))$. Let $K\left(T_{p}(\mathcal{K})\right):=K(B(\mathcal{K})) \cap T_{p}(\mathcal{K})$ for $p \in[1, \infty]$ be a closed pointed generating cone in $S(B(\mathcal{K})) \cap T_{p}(\mathcal{K})$. Recall that $B(\mathcal{K})$ is the dual space of $T_{1}(\mathcal{K})$, where $a(g)=\operatorname{tr}(g a)$ for $a \in B(\mathcal{K})$ and $g \in T_{1}(\mathcal{K})$ [9, Theorem VI.26 (b)] or [13]. The weak star topology $\left(w^{*}\right)$ on $B(\mathcal{K})$ is the weak operator topology, which is also called the ultraweak topology for $C^{*}$ algebras. Recall that $K\left(B(\mathcal{K})\right.$ ) is the dual cone of $K\left(T_{1}(\mathcal{K})\right.$ ). A sequence $a_{n} \in K(B(\mathcal{H})$ ) is called increasing if $0 \leqslant a_{1} \leqslant \cdots \leqslant a_{n} \leqslant \cdots$. Assume that above sequence is bounded by norm. Then there exists a subnet of this sequence that converge in $w^{*}$ topology to $a \in K(B(\mathcal{K}))$ [7]. This is equivalent to the statement that $\lim _{n \rightarrow \infty}=\left\langle a_{n} \mathbf{x}, \mathbf{x}\right\rangle=\langle a \mathbf{x}, \mathbf{x}\rangle$ for each $\mathbf{x} \in \mathcal{K}$. We denote $a=\sup a_{n}$. Assume that $\mu: B(\mathcal{K}) \rightarrow B(\mathcal{H})$ is a bounded positive linear map $(\mu(K(B(\mathcal{K}))) \subset K(B(\mathcal{H}))$. Then $\mu$ is called is called normal if for each increasing seqeunce $a_{n} \in K(B(\mathcal{K}))$ we have the equality $\mu\left(\sup a_{n}\right)=\sup \mu\left(a_{n}\right)$. This is equivalent to the assumptions that every $w^{*}$ convergent net in $B(\mathcal{K})$ is mapped to a $w^{*}$ convergent net in $B(\mathcal{H})[7,11]$. Thus if in Theorem $1 \mu$ is normal the condition $\mu$ maps strongly convergent sequence to weakly convergent sequences are satisfied.

\section{Representation of quantum channels}

Denote by $S\left(B\left(\mathbb{C}^{n}\right)\right)=\mathrm{H}_{n} \supset \mathrm{H}_{n,+}=K\left(B\left(\mathbb{C}^{n}\right)\right) \supset \mathrm{H}_{n,+, 1}$ the real space of $n \times n$ hermitian matrices, the cone of positive (semidefinite) matrices and the convex set of density matrices. Let $\mu: \mathbb{C}^{n \times n} \rightarrow \mathbb{C}^{m \times m}$ be a completely positive map:

$$
\mu(X)=\sum_{i=1}^{k} A_{i} X A_{i}^{*}, \quad A_{i} \in \mathbb{C}^{m \times n}, i \in[k] .
$$

A standard definition of a quantum channel is a completely positive map $\mu$ that preserves density matrices. This is equivalent to the assumption that $\mu$ is trace preserving, i.e. $\sum_{i=1}^{k} A_{i}^{*} A_{i}=I_{n}$ [4]. A more general definiton can be stated as in [5, 8]. We call a nonzero completely positive operator $\mu$ given by (4) a quantum subchannel if $\operatorname{tr} X \geqslant \operatorname{tr} \mu(X)$ for each $X \in \mathrm{H}_{n,+, 1}$. This is equivalent to the assumption, e.g. [4],

$$
0 \lesseqgtr \sum_{i=1}^{k} A_{i}^{*} A_{i} \leqslant I_{n} .
$$

Then the nonlinear transformation $X \mapsto \frac{1}{\operatorname{tr} X} \mu(X)$ is a nonlinear quantum channel, which takes $X \in \mathrm{H}_{n,+, 1}$ to $\frac{1}{\operatorname{tr} X} \mu(X)$ with probability $\operatorname{tr} \mu(X)$.

Let $\mathcal{K}$ be a separable infinite dimensional Hilbert space. Then $g \in T_{1}(\mathcal{K})$ is called a density operator (matrix) if $g \geqslant 0$ and $\operatorname{tr} g=1$. Let $\mathcal{H}$ be another separable infinite dimensional Hilbert space. Then $\mu: T_{1}(\mathcal{K}) \rightarrow T_{1}(\mathcal{H})$ is called quantum channel if $\mu$ is completely positive operator which preserves the density operators. This is equivalent to the assumption that $\mu$ is trace preserving. We call $\mu$ a quantum subchannel if $\mu$ is completely positive operator such that for each density operator $g$ we have $\operatorname{tr} g \geqslant \operatorname{tr} \mu(g)$. The following theorem gives a simple neccessary and sufficient conditions for $\mu: T_{1}(\mathcal{K}) \rightarrow T_{1}(\mathcal{H})$ to be a quantum subchannel. Different necessary and sufficient conditions for trace preserving map $\mu: T_{1}(\mathcal{K}) \rightarrow T_{1}(\mathcal{H})$ are given in [6].

Theorem 7. Let $\mathcal{K}$ and $\mathcal{H}$ be Hilbert spaces with infinite countable bases $\left\{\mathbf{k}_{i}\right\},\left\{\mathbf{h}_{i}\right\}, i \in \mathbb{N}$, respectively. For each $n \in \mathbb{N}$ denote by $P_{n}$ the orthogonal projection of $\mathcal{H}$ on $\operatorname{span}\left(\mathbf{h}_{1}, \ldots, \mathbf{h}_{n}\right)$ Assume that $\mu: T_{1}(\mathcal{K}) \rightarrow T_{1}(\mathcal{H})$ is a bounded linear operator. Then $\mu$ is a subchannel if and only if for each $n \in \mathbb{N}$ the following conditions hold: 
1. The matrix $L_{n}(\mu)=\left[P_{n} \mu\left(\mathbf{k}_{i} \mathbf{k}_{j}^{\vee}\right) P_{n}\right]_{i, j \in[n]} \in B(\mathcal{H})^{(n)}$ is positive.

2. The $n \times n$ matrix $M_{n}:=\left[\operatorname{tr} P_{n} \mu\left(\mathbf{k}_{i} \mathbf{k}_{i}^{\vee}\right) P_{n}\right]_{i, j \in[n]}$ satisfies $M_{n} \leqslant I_{n}$.

Furthermore, $\mu$ is a trace preserving channel if and only if the following conditions hold: First the above condition (1) holds for each $n \in \mathbb{N}$. Second

$$
\operatorname{tr} \mu\left(\mathbf{k}_{i} \mathbf{k}_{j}^{\vee}\right)=\delta_{i j}, \quad i, j \in \mathbb{N} .
$$

Proof. In view of Theorem 1 and Lemma 5 it follows that the condition (1) is a necessary and sufficient conditions for $\mu$ to be completely positive. We now show that if $\mu$ is a quantum subchannel then the condition (2) holds. Suppose that $g \in T_{1}(\mathcal{H})$. Recall that $\operatorname{tr} g=\sum_{i=1}^{\infty}\left\langle g \mathbf{h}_{i}, \mathbf{h}_{i}\right\rangle$. Assume that $g \geqslant 0$. Then

$$
\operatorname{tr} g \geqslant \sum_{i=1}^{n}\left\langle g \mathbf{h}_{i}, \mathbf{h}_{i}\right\rangle=\operatorname{tr} P_{n} g P_{n} .
$$

Assume that $f \in T_{1}(\mathcal{K})$ and $f \geqslant 0$. The assumption that $\mu$ is a subchannel yields $\operatorname{that} \operatorname{tr} f \geqslant \operatorname{tr} \mu(f) \geqslant$ $\operatorname{tr} P_{n} \mu(f) P_{n}$. Choose $f=\mathbf{u u}^{\vee}$, where $\mathbf{u}=\sum_{i=1} x_{i} \mathbf{k}_{i}$. Let $\mathbf{x}=\left(x_{1}, \ldots, x_{n}\right)^{\top}$. Thus $\operatorname{tr} f=\mathbf{x}^{*} \mathbf{x}$ and $\operatorname{tr} P_{n} \mu(f) P_{n}=$ $\mathbf{x}^{*} M_{n} \mathbf{x}$. Hence $M_{n} \leqslant I_{n}$.

It is left to show that if the condition (2) is sufficient for a completely positive map to be a quantum subchannel. Fix $n \in \mathbb{N}$ and let $m \geqslant n$. Let $f=\mathbf{u u}^{\vee}$ be defined as above. We then obtain $\operatorname{that} \operatorname{tr} f \geqslant \operatorname{tr} P_{m} \mu(f) P_{m}$. Use the arguments of the proof of Theorem 1 to deduce that $\operatorname{tr} f \geqslant \operatorname{tr} \mu(f)$. Assume that $a \in T_{1}(\mathcal{K}), a \geqslant 0$ and the range of $a$ is contained in $\operatorname{span}\left(\mathbf{k}_{1}, \ldots, \mathbf{k}_{n}\right)$. Then $a=\sum_{j=1}^{n} \mathbf{u}_{j} \mathbf{u}_{j}^{\vee}$ for some $\mathbf{u}_{1}, \ldots, \mathbf{u}_{n} \in \operatorname{span}\left(\mathbf{k}_{1}, \ldots, \mathbf{k}_{n}\right)$. Hence

$$
\operatorname{tr} a=\sum_{j=1}^{n} \operatorname{tr} \mathbf{u}_{j} \mathbf{u}_{j}^{\vee} \geqslant \sum_{j=1}^{n} \operatorname{tr} \mu\left(\mathbf{u}_{j} \mathbf{u}_{j}^{\vee}\right)=\operatorname{tr} \mu(a) .
$$

Let $b \in T_{1}(\mathcal{K}), b \geqslant 0$. Then $P_{n} b P_{n}$ is of the above form and we get that $\operatorname{tr} P_{n} b P_{n} \geqslant \operatorname{tr} \mu\left(P_{n} b P_{n}\right)$. Let $n \rightarrow \infty$ and use Lemma 5 to deduce that $\operatorname{tr} b \geqslant \operatorname{tr} \mu(b)$.

It is left to discuss the necessary and sufficient conditions for a completely positive $\mu$ to be trace preserving. Suppose first that $\mu$ is trace preserving. Clearly, condition (1) holds. As $\mu$ is trace preserving and $\operatorname{tr} \mathbf{k}_{i} \mathbf{k}_{j}^{\vee}=\left\langle\mathbf{k}_{i}, \mathbf{k}_{j}\right\rangle=\delta_{i j}$ we deduce that the condition (6) holds. It is left to show that the condition (1) and (6) yield that $\mu$ is quantum channel. As in the previous case the condition (1) ensures that $\mu$ is completely positive. It is left to show that $\mu$ is trace preserving. Assume as above that $a \geqslant 0$ and range of $a$ is contained in $\operatorname{span}\left(\mathbf{k}_{1}, \ldots, \mathbf{k}_{n}\right)$. The the condition (6) yields that $\operatorname{tr} a=\operatorname{tr} \mu(a)$. Use the above arguments for $b \geqslant 0$ to deduce that $\operatorname{tr} b=\operatorname{tr} \mu(b)$. As every selfadjoint $b \in T_{1}(\mathcal{K})$ is of the form $b_{+}-b_{-}$, where $b_{+}, b_{-} \in T_{-}(\mathcal{K})$ and $b_{+}, b_{-} \geqslant 0$ it follows that $\operatorname{tr} b=\operatorname{tr} \mu(b)$ for each selfadjoint in $T_{1}(\mathcal{K})$. As $\mu$ is linear it follows that $\mu$ is trace preserving on $T_{1}(\mathcal{K})$.

Recall that the dual space of $T_{1}(\mathcal{H})$, denoted as $T_{1}(\mathcal{H})^{\vee}$, is $B(\mathcal{H})$. Assume that $\mu: T_{1}(\mathcal{K}) \rightarrow T_{1}(\mathcal{H})$ be a bounded linear operator. Denote by $\mu^{\vee}$ the induced linear operator $\mu^{\vee}: B(\mathcal{H}) \rightarrow B(\mathcal{K})$.

Theorem 8. Let $\mathcal{K}$ and $\mathcal{H}$ be Hilbert spaces with infinite countable bases $\left\{\mathbf{k}_{i}\right\},\left\{\mathbf{h}_{i}\right\}, i \in \mathbb{N}$, respectively. Assume that the bounded linear operator $\mu: T_{1}(\mathcal{K}) \rightarrow T_{1}(\mathcal{H})$ is completely positive. Then the map $\mu^{\vee}: B(\mathcal{H}) \rightarrow$ $B(\mathcal{K})$ is completely positive.

Proof. As $\mu$ is completely positive it follows that $\mu$ maps seladjoint operators to selfadjoint. Hence $\mu\left(a^{*}\right)=$ $\mu(a)^{*}$. Therefore $\mu^{\vee}\left(b^{*}\right)=\left(\mu^{\vee}(b)\right)^{*}$. We now apply Theorem 1 . Thus $\mathcal{H}_{1}=\mathcal{H}, \mathcal{H}_{2}=\mathcal{K}, \Sigma_{1}=B(\mathcal{H}), \Sigma_{2}=B(\mathcal{K})$.

We first observe that condition (4) holds. Let $P_{n}$ be the orthogonal projection of $\mathcal{H}$ on $\operatorname{span}\left(\mathbf{h}_{1}, \ldots, \mathbf{h}_{n}\right)$. Assume that $b \in B(\mathcal{H})$. We need to show that $\lim _{n \rightarrow \infty}\left\langle\mu^{\vee}\left(P_{n} b P_{n}\right) \mathbf{x}, \mathbf{y}\right\rangle=\left\langle\mu^{\vee}(b) \mathbf{x}, \mathbf{y}\right\rangle$ for each pair $\mathbf{x}, \mathbf{y} \in \mathcal{K}$. Clearly

$$
\left\langle\mu^{\vee}\left(P_{n} b P_{n}\right) \mathbf{x}, \mathbf{y}\right\rangle=\operatorname{tr} \mu^{\vee}\left(P_{n} b P_{n}\right) \mathbf{x y} \mathbf{y}^{\vee}=\operatorname{tr} P_{n} b P_{n} \mu\left(\mathbf{x y} \mathbf{y}^{\vee}\right) .
$$


Write down the singular value decomposition of $\mu\left(\mathbf{x y}^{\vee}\right) \in T_{1}(\mathcal{H})$ :

$$
\mu\left(\mathbf{x y}^{\vee}\right)=\sum_{i=1}^{\infty} \sigma_{i} \mathbf{u}_{i} \mathbf{v}_{i}^{\vee}, \quad \sum_{i=1}^{\infty} \sigma_{i}<\infty,\left\langle\mathbf{u}_{i}, \mathbf{u}_{j}\right\rangle=\left\langle\mathbf{v}_{i}, \mathbf{v}_{j}\right\rangle=\delta_{i j}, i, j \in \mathbb{N} .
$$

Then

$$
\operatorname{tr} P_{n} b P_{n} \mathbf{u}_{i} \mathbf{v}_{i}^{\vee}=\left\langle P_{n} b P_{n} \mathbf{u}_{i}, \mathbf{v}_{i}\right\rangle,\left|\operatorname{tr} P_{n} b P_{n} \mathbf{u}_{i} \mathbf{v}_{i}^{\vee}\right| \leqslant\|b\|, \quad \lim _{n \rightarrow \infty} \operatorname{tr} P_{n} b P_{n} \mathbf{u}_{i} \mathbf{v}_{i}^{\vee}=\operatorname{tr} b \mathbf{u}_{i} \mathbf{v}_{i}^{\vee}, \quad i \in \mathbb{N} .
$$

Therefore

$$
\lim _{n \rightarrow \infty}\left\langle\mu^{\vee}\left(P_{n} b P_{n}\right) \mathbf{x}, \mathbf{y}\right\rangle=\sum_{i=1}^{\infty} \sigma_{i}\left(\operatorname{tr} b \mathbf{u}_{i} \mathbf{v}_{i}^{\vee}\right)=\operatorname{tr} b \mu\left(\mathbf{x y}^{\vee}\right)=\left\langle\mu^{\vee}(b) \mathbf{x}, \mathbf{y}\right\rangle .
$$

Let $Q_{n}: \mathcal{K} \rightarrow \mathcal{K}$ be the orthogonal projection on $\operatorname{span}\left(\mathbf{k}_{1}, \ldots, \mathbf{k}_{n}\right)$. It is left to show that for each $n \in \mathbb{N}$ the matrix $L_{n}\left(\mu^{\vee}\right)=\left[Q_{n} \mu^{\vee}\left(\mathbf{h}_{i} \mathbf{h}_{j}^{\vee}\right) Q_{n}\right]_{i, j \in[n]} \in B(\mathcal{K})^{(n)}$ is positive. That is,

$$
0 \leqslant \sum_{i, j=1}^{n}\left\langle\mu^{\vee}\left(\mathbf{h}_{i} \mathbf{h}_{j}^{\vee}\right) \mathbf{x}_{i}, \mathbf{x}_{j}\right\rangle=\sum_{i, j=1}^{n} \operatorname{tr} \mu^{\vee}\left(\mathbf{h}_{i} \mathbf{h}_{j}^{\vee}\right) \mathbf{x}_{i} \mathbf{x}_{j}^{\vee}=\sum_{i, j=1}^{n} \operatorname{tr} \mathbf{h}_{i} \mathbf{h}_{j}^{\vee} \mu\left(\mathbf{x}_{i} \mathbf{x}_{j}^{\vee}\right)=\sum_{i, j=1}^{n}\left\langle\mu\left(\mathbf{x}_{i} \mathbf{x}_{j}^{\vee}\right) \mathbf{h}_{i}, \mathbf{h}_{j}\right\rangle
$$

for a given $\mathbf{x}_{1}, \ldots, \mathbf{x}_{n} \in \operatorname{span}\left(\mathbf{k}_{1}, \ldots, \mathbf{k}_{n}\right)$. The above inequality follows from the assumption that the map $\mu: T_{1}(\mathcal{K}) \rightarrow T_{1}(\mathcal{H})$ is completely positive.

Following the papers of Hellwig-Kraus [5] and Kraus [8] we explain how to generate special quantum subchannel $\mu: T_{1}(\mathcal{K}) \rightarrow T_{1}(\mathcal{H})$ using the "trace out" notion. We consider two quantum systems which are represented as density operators $a \in T_{1}(\mathcal{K})$ and $b \in T_{1}(\mathcal{H})$. The combined system is described by $a \otimes b \in T_{1}(\mathcal{K} \otimes \mathcal{H})$. The combined system evolves to a new system $c=U(a \otimes b) U^{*}$, where $U$ is a unitary operator on $\mathcal{K} \otimes \mathcal{H}$. Let $Q: \mathcal{K} \rightarrow \mathcal{K}$ be an orthogonal projection. Denote $\hat{\mathcal{K}}=Q \mathcal{K}$. Assume that $\hat{\mathbf{k}}_{i}, i \in[N]$ is an orthonormal basis of $\hat{\mathcal{K}}$. (Here either $N \in \mathbb{N}$ or $N=\infty$ and $[\infty]=\mathbb{N}$.) Let $e=(Q \otimes I d) d(Q \otimes I d) \in T_{1}\left(\mathcal{K}_{1} \otimes \mathcal{H}\right)$. Note that $e \geqslant 0$ and tr $e \leqslant 1$. Denote by

$$
\operatorname{tr}_{\hat{\mathcal{K}}}(e)=\sum_{i=j=1}^{\infty}\left(\sum_{p \in[N]}\left\langle e\left(\hat{\mathbf{k}}_{p} \otimes \mathbf{h}_{i}\right), \hat{\mathbf{k}}_{p} \otimes \mathbf{h}_{j}\right\rangle\right) \mathbf{h}_{i} \mathbf{h}_{j}^{\vee} .
$$

This is the "trace out" operation. It is well known that $\operatorname{tr}_{\hat{\mathcal{K}}}(e) \in T_{1}(\mathcal{H}), e \geqslant 0$, and $\operatorname{tr}\left(\operatorname{tr}_{\hat{\mathcal{K}}}(e)\right)=\operatorname{tr} e$. Then we obtain a new system $\mu(a)=\operatorname{tr}_{\hat{\mathcal{K}}}(e) \in T_{1}(\mathcal{H})$. As in [5] it follows that

$$
\mu(a)=\sum_{i=1}^{\infty} A_{i} a A_{i}^{\vee}, A_{i} \in L(\mathcal{K}, \mathcal{H}), i \in N, \quad \sum_{i=1}^{\infty} A_{i}^{\vee} A_{i} \leqslant I d .
$$

For $\hat{\mathcal{K}}=\mathcal{K}$ we get the equality $\sum_{i=1}^{\infty} A_{i}^{\vee} A_{i}=I d$.

Note that the convergence of the infinite sum in (7) is in the weak operator topology. Furthermore, it is straightforward to show that if $\mu$ is a quantum subchannel given by (7) then the dual $\mu^{\vee}: B(\mathcal{K}) \rightarrow B(\mathcal{H})$ is given by $\mu^{\vee}(c)=\sum_{i=1}^{\infty} A_{i}^{\vee} c A_{i}$.

It is shown in [6] that every trace preserving $\mu: T_{1}(\mathcal{K}) \rightarrow T_{1}(\mathcal{H})$, where $\mathcal{K}$ and $\mathcal{H}$ are separable, has the Krauss representation (7) with $\sum_{i=1}^{\infty} A_{i}^{\vee} A_{i}=I d$. We now use the arguments in $[6,8]$ to show that any quantum subchannel $\mu: \mathcal{K} \rightarrow \mathcal{H}$ has the form (7). For $\mathcal{K}=\mathcal{H}$ this result is [8, Theorem 3.3] under the additional assumption that $\mu$ is continuous in the weak operator topology.

Theorem 9. Let $\mathcal{K}$ and $\mathcal{H}$ be two separable Hilbert spaces. Then $\mu: \mathcal{K} \rightarrow \mathcal{H}$ is a quantum subchannel if and only if there exist bounded linear operators $A_{i} \in L(\mathcal{K}, \mathcal{H})$ for $i \in \mathbb{N}$ such that (7) holds,

Proof. Assume that $\mu: \mathcal{K} \rightarrow \mathcal{H}$ is a quantum subchannel. Let $\mathcal{K} \circ \mathcal{H}^{\vee}$ be the algebraic tensor product of $\mathcal{K}$ and $\mathcal{H}^{\vee}$, (the dual space of $\mathcal{H}$ ). That is, the elements of $\mathcal{K} \circ \mathcal{H}^{\vee}$ are finite sums of the form $\sum_{i=1}^{N} \mathbf{x}_{i} \otimes \mathbf{u}_{i}^{\vee}$ for $\mathbf{x}_{i} \in \mathcal{K}, \mathbf{u}_{i} \in \mathcal{H}, N \in \mathbb{N}$. We define the following sesquilinear form on $\mathcal{K} \circ \mathcal{H}:$

$$
\Omega_{\mu}\left(\sum_{i=p=1}^{N} \mathbf{x}_{i} \otimes \mathbf{u}_{p}^{\vee}, \sum_{j=q=1}^{N} \mathbf{y}_{j} \otimes \mathbf{v}_{q}^{\vee}\right)=\sum_{i=j=p=q=1}^{N}\left\langle\mu\left(\mathbf{x}_{i} \mathbf{y}_{j}^{\vee}\right) \mathbf{v}_{q}, \mathbf{u}_{p}\right\rangle .
$$


The assumption that $\mu$ is completely positive means that $\Omega_{\mu}$ is positive semidefinite. Then $\Omega_{\mu}$ defines a semiinner product and a seminorm

$$
\langle\mathbf{z}, \mathbf{w}\rangle_{\mu}=\Omega_{\mu}(\mathbf{z}, \mathbf{w}), \quad\|\mathbf{z}\|_{\mu}=\sqrt{\Omega_{\mu}(\mathbf{z}, \mathbf{z})}, \quad \mathbf{z}, \mathbf{w} \in \mathcal{K} \circ \mathcal{H}^{\vee} .
$$

Clearly, we have the Cauchy-Schwarz inequality $\left|\langle\mathbf{z}, \mathbf{w}\rangle_{\mu}\right| \leqslant\|\mathbf{z}\|_{\mu}\|\mathbf{w}\|_{\mu}$. Let $\mathcal{L}$ be the closure of $\mathcal{K} \circ \mathcal{H}^{\vee}$ with respect to the semimetric $\|\cdot\|_{\mu}$. Then we can extend the semiinner product $\langle\cdot, \cdot\rangle_{\mu}$ and the seminorm $\|\cdot\|_{\mu}$ to $\mathcal{L}$. Denote by $\mathcal{M}$ the linear subspace of all $\mathbf{z} \in \mathcal{L}$ such that $\|\mathbf{z}\|_{\mu}=0$. Then $\mathcal{N}=\mathcal{L} / \mathcal{M}$ is a separable Hilbert space. Assume for simplicity of the exposition that $\mathcal{N}$ is infinite dimensional. Let $\mathbf{f}_{l}, l \in \mathbb{N}$ be an orthonormal basis of $\mathcal{N}$. For $l \in \mathbb{N}$ denote by $\phi_{l}: \mathcal{N} \rightarrow \mathbb{C}$ the linear functional $\phi_{l}(\mathbf{z})=\left\langle\mathbf{z}, \mathbf{f}_{l}\right\rangle_{\mu}$. Then $\phi_{l}$ extends to a linear functional $\hat{\phi}_{l}: \mathcal{M} \rightarrow \mathbb{C}: \hat{\phi}_{l}(\mathbf{w})=\left\langle\mathbf{w}, \mathbf{f}_{l}\right\rangle_{\mu}$ and $\left|\left\langle\mathbf{w}, \mathbf{f}_{l}\right\rangle_{\mu}\right| \leqslant\|\mathbf{w}\|_{\mu}$. Moreover

$$
\mathbf{w}=\sum_{l=1}^{\infty}\left\langle\mathbf{w}, \mathbf{f}_{l}\right\rangle_{\mu} \mathbf{f}_{l}, \quad\|\mathbf{w}\|_{\mu}^{2}=\sum_{l=1}^{\infty}\left|\left\langle\mathbf{w}, \mathbf{f}_{l}\right\rangle_{\mu}\right|^{2}, \mathbf{w} \in \mathcal{M} .
$$

In particular

$$
\left\|\mathbf{x} \otimes \mathbf{u}^{\vee}\right\|_{\mu}^{2}=\left\langle\mu\left(\mathbf{x} \mathbf{x}^{\vee}\right) \mathbf{u}, \mathbf{u}\right\rangle=\sum_{l=1}^{\infty}\left|\left\langle\mathbf{x} \otimes \mathbf{u}^{\vee}, \mathbf{f}_{l}\right\rangle_{\mu}\right|^{2} .
$$

Assume that $\mathbf{u} \neq 0$ and let $\mathbf{u}_{i}, i \in \mathbb{N}$ be an orthonormal basis, where $\mathbf{u}_{1}=\frac{1}{\|\mathbf{u}\|} \mathbf{u}$. Then $\operatorname{tr} \mu\left(\mathbf{x x}^{\vee}\right)=$ $\sum_{i=1}^{\infty}\left\langle\mu\left(\mathbf{x} \mathbf{x}^{\vee}\right) \mathbf{u}_{i}, \mathbf{u}_{i}\right\rangle$. As $\mu$ is subchannel we obtain

$$
\sum_{i=1}^{\infty}\left\langle\mu\left(\mathbf{x} \mathbf{x}^{\vee}\right) \mathbf{u}_{i}, \mathbf{u}_{i}\right\rangle \leqslant \operatorname{tr} \mathbf{x} \mathbf{x}^{\vee}=\|\mathbf{x}\|^{2} .
$$

In particular $\left\|\mathbf{x} \otimes \mathbf{u}^{\vee}\right\|_{\mu} \leqslant\|\mathbf{x}\|\|\mathbf{u}\|$. Define $A_{l}: \mathcal{K} \rightarrow \mathcal{H}$ by equality $\left\langle A_{l} \mathbf{x}, \mathbf{u}\right\rangle=\left\langle\mathbf{x} \otimes \mathbf{u}^{\vee}, \mathbf{f}_{l}\right\rangle_{\mu}$. So $\left\|A_{l}\right\| \leqslant 1$ and $A_{l} \mathbf{x}=\sum_{i=1}^{\infty}\left\langle\mathbf{x} \otimes \mathbf{u}_{i}^{\vee}, \mathbf{f}_{l}\right\rangle_{\mu} \mathbf{u}_{i}$. Thus

$$
\begin{array}{r}
\left\langle\left(\sum_{l=1}^{N} A_{l}^{\vee} A_{l}\right) \mathbf{x}, \mathbf{x}\right\rangle=\sum_{l=1}^{N}\left\langle A_{l}^{\vee} A_{l} \mathbf{x}, \mathbf{x}\right\rangle=\sum_{l=1}^{N}\left\langle A_{l} \mathbf{x}, A_{l} \mathbf{x}\right\rangle=\sum_{l=1}^{N}\left\langle\sum_{i=1}^{\infty}\left\langle\mathbf{x} \otimes \mathbf{u}_{i}^{\vee}, \mathbf{f}_{l}\right\rangle_{\mu} \mathbf{u}_{i}, \sum_{j=1}^{\infty}\left\langle\mathbf{x} \otimes \mathbf{u}_{j}^{\vee}\right\rangle_{\mu} \mathbf{u}_{j}\right\rangle= \\
\sum_{l=1}^{N} \sum_{i=1}^{\infty}\left|\left\langle\mathbf{x} \otimes \mathbf{u}_{i}^{\vee}, \mathbf{f}_{l}\right\rangle_{\mu}\right|^{2}=\sum_{i=1}^{\infty} \sum_{l=1}^{N}\left|\left\langle\mathbf{x} \otimes \mathbf{u}_{i}^{\vee}, \mathbf{f}_{l}\right\rangle_{\mu}\right|^{2} \leqslant \sum_{i=1}^{\infty}\left\langle\mu\left(\mathbf{x x}^{\vee}\right) \mathbf{u}_{i}, \mathbf{u}_{i}\right\rangle \leqslant \operatorname{tr} \mathbf{x} \mathbf{x}^{\vee}=\|\mathbf{x}\|^{2} .
\end{array}
$$

Thus $\sum_{l=1}^{N} A_{l}^{\vee} A_{l} \leqslant I d$ for each $N \in \mathbb{N}$. Hence $\sum_{l=1}^{\infty} A_{l}^{\vee} A_{l} \leqslant I_{d}$.

It is left to show that $\mu$ is given by (7). Let $\mathbf{x}, \mathbf{y} \in \mathcal{K}$. Then $\mathbf{x y}^{\vee} \in T_{1}(\mathcal{K})$. We need to show that $\mu\left(\mathbf{x y} \mathbf{y}^{\vee}\right)=$ $\sum_{l=1}^{\infty}\left(A_{l} \mathbf{x}\right)\left(A_{l} \mathbf{y}\right)^{\vee}$. Thus, it is enough to show that

$$
\left\langle\mu\left(\mathbf{x y}^{\vee}\right) \mathbf{u}, \mathbf{v}\right\rangle=\sum_{l=1}^{\infty}\left\langle\mathbf{u}, A_{l} \mathbf{y}\right\rangle\left\langle A_{l} \mathbf{x}, \mathbf{v}\right\rangle \quad \text { for all } \mathbf{x}, \mathbf{y} \in \mathcal{K}, \mathbf{u}, \mathbf{v} \in \mathcal{H} .
$$

Recall that $\left\langle A_{l} \mathbf{x}, \mathbf{v}\right\rangle=\left\langle\mathbf{x} \otimes \mathbf{v}^{\vee}, \mathbf{f}_{l}\right\rangle_{\mu}$ and $\left\langle\mathbf{u}, A_{l} \mathbf{y}\right\rangle=\overline{\left\langle A_{l} \mathbf{y}, \mathbf{u}\right\rangle}=\overline{\left\langle\mathbf{y} \otimes \mathbf{u}^{\vee}, \mathbf{f}_{l}\right\rangle_{\mu}}$. Hence

$$
\sum_{l=1}^{\infty}\left\langle\mathbf{u}, A_{l} \mathbf{y}\right\rangle\left\langle A_{l} \mathbf{x}, \mathbf{v}\right\rangle=\sum_{l=1}^{\infty}\left\langle\mathbf{x} \otimes \mathbf{v}^{\vee}, \mathbf{f}_{l}\right\rangle_{\mu} \overline{\left\langle\mathbf{y} \otimes \mathbf{u}^{\vee}, \mathbf{f}_{l}\right\rangle_{\mu}}=\left\langle\mathbf{x} \otimes \mathbf{v}^{\vee}, \mathbf{y} \otimes \mathbf{u}^{\vee}\right\rangle_{\mu}=\left\langle\mu\left(\mathbf{x y} \mathbf{y}^{\vee}\right) \mathbf{u}, \mathbf{v}\right\rangle .
$$

Assume that (7) holds. For $N \in \mathbb{N}$ define $\mu_{N}: B(\mathcal{K}) \rightarrow B(\mathcal{H})$ by the equality $\mu_{N}(a)=\sum_{l=1}^{N} A_{l} a A_{l}^{\vee}$. Clearly, $\mu_{N}$ is completely positive. Also $\mu_{N}$ is a quantum subchannel as $\mu_{N}: T_{1}(\mathcal{K}) \rightarrow T_{1}(\mathcal{H})$. Therefore $\mu$ given by $(7)$ is a subchannel.

\section{References}

[1] M.D. Choi, Completely positive linear maps on complex matrices, Linear Algebra Appl. 10 (1975), 285-290. 
[2] K.R. Davidson, C*-algebras by example, Fields Institute Monographs, vol. 6, American Mathematical Society, Providence, RI, 1996.

[3] R.S. Doran and V.A. Belfi, Characterizations of C*-algebras: The Gelfand-Naimark Theorems, CRC Press, 1986.

[4] S. Friedland and R. Loewy, On the extreme points of quantum channels, Linear Algebra Appl. 498 (2016), 553-573.

[5] K.-E. Hellwig and K. Kraus, Pure operations and measurements, Commun. Math. Phys., 11 (1969), 214-220.

[6] A. S. Holevo, Entropy gain and the Choi-Jamiolkowski correspondence for infinite-dimensional quantum evolutions, Theor. Math. Phys. 166 (2011), no. 1, 123-138.

[7] S. Karlin, Positive operators, J. Math. Mech. 8 (1959), 907-937.

[8] K. Kraus, General state changes in quantum theory, Ann. Phys. 64 (1971), 311-335.

[9] M. Reed and B. Simon, Methods of Modern Mathematical Physics: Functional Analysis I, Academic Press, 1998.

[10] M. Rørdam, Classification of Nuclear, Simple C*-algebras, Classification of Nuclear C*-Algebras. Entropy in Operator Algebras (J. Cuntz and V. Jones, eds.), vol. 126, Encyclopaedia of Mathematical Sciences. Subseries: Operator Algebras and Noncommutative Geometry, no. VII, Springer Verlag, Berlin, Heidelberg, 2001, pp. 1-145.

[11] S. Sakai, $C^{*}$-algebras and $W^{*}$-algebras, Springer 1971.

[12] R. Schatten, Norm Ideals of Completely Continuous Operators, Springer- Verlag, Berlin, 1960.

[13] B. Simon, Trace ideals and their applications, Second Edition, Amer. Math. Soc. 2005.

[14] W. F. Stinespring, Positive functions on $C^{*}$-algebras, Proc. Amer. Math. Soc. 6 (1955), 211-216.

[15] E. Størmer, The analogue of Choi matrices for a class of linear maps on Von Neumann algebras, Internat. J. Math. 26 (2015), no. 2, 1550018, 7 pp.

[16] J. Watrous, The theory of quantum information, Cambridge University Press, 2018. 